\title{
Erratum to: Simultaneous Qualitative Analysis of Coumarins in the Rhizome of Angelica polymorpha Maxim. by LC-DAD-ESI-MS
}

\author{
Na Liu • Nengjiang Yu $\cdot$ Jin Li $\cdot$ Yu Yang • \\ Jifen Guo • Yimin Zhao
}

Published online: 29 May 2011

(C) Springer-Verlag 2011

Erratum to: Chromatographia (2011) 73:1121-1129

DOI 10.1007/s10337-011-2008-4

Unfortunately, in the original article names of the authors N. J. Yu, J. F. Guo and Y. M. Zhao were given incorrectly. The correct names are Nengjiang Yu, Jifen Guo and Yimin Zhao.

The online version of the original article can be found under doi:10.1007/s10337-011-2008-4.

N. Liu $\cdot$ N. Yu $(\bowtie) \cdot$ Y. Yang · J. Guo $~$ Y. Zhao Beijing Institute of Pharmacology and Toxicology, Beijing 100850, People's Republic of China e-mail: ynj08@hotmail.com

N. Liu $\cdot$ J. Li

Tianjin University of Traditional Chinese Medicine,

Tianjin 300193, People's Republic of China 